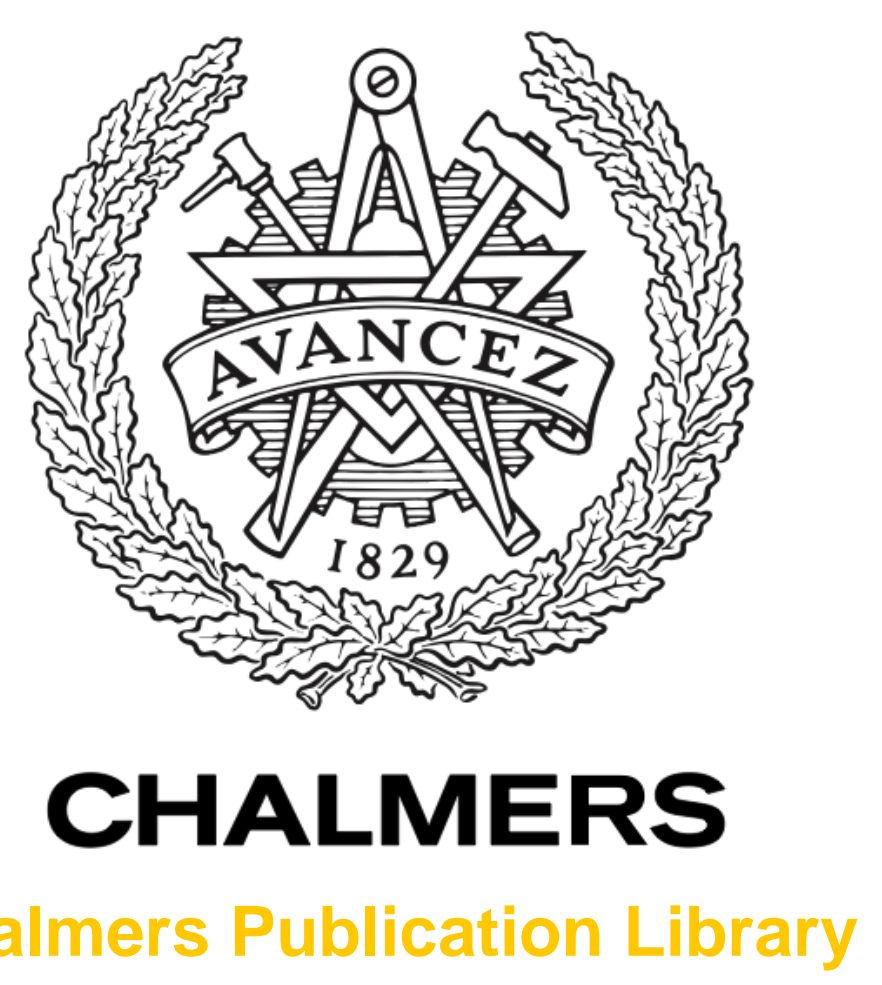

Chalmers Publication Library

\title{
Streamer Propagation in Hybrid Gas-Solid Insulation
}

This document has been downloaded from Chalmers Publication Library (CPL). It is the author's version of a work that was accepted for publication in:

2015 Annual Report of IEEE Conference on Electric Insulation and Dielectric Phenomena, CEIDP 2015, Ann Arbor, United States, 18-21 October 2015 (ISSN: 0084-9162)

Citation for the published paper:

Singh, S. ; Serdyuk, Y. ; Summer, R. (2015) "Streamer Propagation in Hybrid Gas-Solid Insulation". 2015 Annual Report of IEEE Conference on Electric Insulation and Dielectric Phenomena, CEIDP 2015, Ann Arbor, United States, 18-21 October 2015 pp. 387-390.

http://dx.doi.org/10.1109/CEIDP.2015.7352030

Downloaded from: http://publications.lib.chalmers.se/publication/224875

Notice: Changes introduced as a result of publishing processes such as copy-editing and formatting may not be reflected in this document. For a definitive version of this work, please refer to the published source. Please note that access to the published version might require a subscription.

Chalmers Publication Library (CPL) offers the possibility of retrieving research publications produced at Chalmers University of Technology. It covers all types of publications: articles, dissertations, licentiate theses, masters theses, conference papers, reports etc. Since 2006 it is the official tool for Chalmers official publication statistics. To ensure that Chalmers research results are disseminated as widely as possible, an Open Access Policy has been adopted.

The CPL service is administrated and maintained by Chalmers Library. 


\section{Streamer Propagation in Hybrid Gas-Solid Insulation}

\author{
Shailendra Singh ${ }^{1,2}$, Yuriy V. Serdyuk \\ ${ }^{1}$ Chalmers University of Technology \\ Gothenburg, Sweden \\ singhs@chalmers.se
}

\author{
Raimund Summer \\ ${ }^{2}$ Schneider Electric Sachsenwerk GmbH \\ Rathenausstrasse 2 \\ Regensburg, Germany
}

\begin{abstract}
The influence of a solid dielectric barrier on development of an electrical discharge in air between needle and plane electrodes is analyzed by means of computer simulations. The computational model describing formation and propagation of a streamer in atmospheric air and accounting for charge transport and trapping on barrier surfaces is presented. The results of the simulations performed for $5 \mathrm{~cm}$ air gap containing solid barrier (plate) inserted between the needle and plane electrodes are discussed focusing on discharge dynamics and associated electric fields.
\end{abstract}

Keywords-high voltage insulation, gaseous dielectrics, gas discharge, low temperature plasma, streamer propagation, space charge, dielectric barrier, surface charge, electric field.

\section{INTRODUCTION}

It is known that withstand performance of gaseous highvoltage insulation can be enhanced by introducing solid insulating elements, in particular, dielectric barriers. Such components being placed between energized electrodes play a role of mechanical obstacles for electrical discharges propagating in gas phase and can successfully suppress their development. Introduction of solid barriers, however, makes design of gas-insulated high voltage systems more complex. Moreover, traditionally used semi-empirical design criteria based on evaluations of breakdown conditions of gases are hardly applicable for hybrid gas-solid insulation [1]. Therefore, more fundamental approaches need to be introduced in order to analyze and predict performance of gaseous dielectric media containing solid elements.

To evaluate withstand ability of hybrid system, development of an electrical discharge in gas and its interaction with solid components needs to be analyzed starting from inception stage and up to breakdown. As known, gas discharge is initiated by free electrons which are accelerated in an applied field and gain energy sufficient to cause impact ionization of neutral molecules. This process leads to formation of electron avalanches which may become strong enough (i.e., contain high number of electrons and ionic species) to induce field with the magnitude comparable with the external one. If such threshold in space charge density is reached, an avalanche transforms into a streamer which is essentially a filamentary low-temperature plasma channel capable to propagate for long distances in neutral gas being supported by own field. In air at atmospheric (and higher) pressure, not too large insulating gaps (not exceeding few tens of centimeters) and voltages close to breakdown threshold, discharges appear mostly in form of streamers. They build up relatively conducting paths between energized electrodes thus preceding complete electrical breakdown. In case of a hybrid system, streamer meets surface of dielectric and deposits certain amount of charged species on gas-solid interface thus creating surface charge layer. Further development is dependent upon nature of solid material and conditions on its surface (density of traps, their depth, etc.) as well as in gas in its vicinity. A part of the charge delivered by streamer can be injected into material bulk and stimulate intrinsic conduction processes. Remaining charges may move along the interface being driven by a tangential component of the field enhanced by deposited surface charges. Depending on configuration and dimensions of solid insulating element as well as energy supplied to the discharge channel, streamer can be terminated or progress further until a conductive path between electrodes along solid surface is successfully completed leading to a breakdown.

Charge transport under strong electric fields has been studied by means of computer simulations separately for solid and gas media, see e.g. [2-4] and [5, 6], respectively. Moreover, attempts have been undertaken to link processes in gas and on gas-solid interface while treating the latter as blocking for charge carriers and solid material as an ideal insulator [7, 8]. It is evident, however, that a consistent description of the interaction of streamers with solid insulating surfaces requires simultaneous consideration of charge transport in both phases. Therefore in the present study, computer simulations were conducted combining charge dynamics in a streamer discharge in atmospheric pressure air with charge transport in the bulk and on the surface of a floating solid dielectric barrier placed between energized needle and plane electrodes.

\section{THE MODEL}

To describe charge transport controlled by electric field in both materials (gaseous and solid), so-called drift-diffusion (or hydro-dynamic) approximation is utilized. Within this approach, charge carriers of each type are treated as charged fluids and variations of their densities in time and space are controlled by their drift in the electric filed, diffusion due to gradients of concentrations, rates of generation and losses. The set of particular physical processes to be considered and corresponding rate constants are specific for each material and are defined by its nature. Mathematically, the transport process is described by a set of partial differential equations (one for each type of charge carriers involved) representing conservation of masses of charged species and continuity of their fluxes (i.e. currents). These equations are to be coupled 
with Poisson's equations for electric potential which allows for obtaining electric field distribution needed for calculating local parameters of the processes.

\section{A. Model of Streamer Discharge in Air}

Since kinetics of particular kind of charge carriers is not of interest, three types of species are considered, namely, electrons and generic positive and negative ions. The mass conservation equations are written in terms of densities $N, \mathrm{~m}^{-3}$ :

$\partial N_{\mathrm{e}, \mathrm{p}, \mathrm{n}} / \partial t+\nabla \cdot\left(-N_{\mathrm{e}, \mathrm{p}, \mathrm{n}} \mathbf{w}_{\mathrm{e}, \mathrm{p}, \mathrm{n}}-D_{\mathrm{e}, \mathrm{p}, \mathrm{n}} \nabla N_{\mathrm{e}, \mathrm{p}, \mathrm{n}}\right)=R_{\mathrm{e}, \mathrm{p}, \mathrm{n}}$

Here the subscripts $e, p$ and $n$ represent respectively electrons, positive ions and negative ions; $t$ stands for time; $D$ is diffusion coefficient, $\mathrm{m}^{2} / \mathrm{s}$. Drift velocity vector is defined as $\mathbf{w}=\mu \mathbf{E}$, where $\mu$ is the mobility, $\mathrm{m}^{2} / \mathrm{Vs}$, and $\mathbf{E}$ is the vector of the electric filed, $\mathrm{V} / \mathrm{m}$, obtained from Poisson's equation

$\nabla \cdot\left(\varepsilon_{0} \varepsilon_{\mathrm{r}} \nabla V\right)=-q\left(N_{p}-N_{e}-N_{n}\right), \mathbf{E}=-\nabla \cdot V$

Here, $V$ is the electric potential, $\mathrm{V} ; \varepsilon_{0}$ and $\varepsilon_{\mathrm{r}}$ are permittivity of vacuum, $\mathrm{F} / \mathrm{m}$, and relative permittivity, respectively; $q$ is the elementary charge, $\mathrm{C}$.

The terms $R, \mathrm{~m}^{-3} \mathrm{~s}^{-1}$, on the right hand side of equations (1)(3) represent net rates of generation/loss of species of particular type. In the present study, the rates are

$$
\begin{aligned}
& R_{e}=R_{\text {ion }}+R_{\text {det }}+R_{0}+R_{p h}-R_{a t t}-R_{e p} \\
& R_{p}=R_{\text {ion }}+R_{0}+R_{p h}-R_{e p}-R_{p n} \\
& R_{n}=R_{\text {att }}-R_{\text {det }}-R_{p n}
\end{aligned}
$$

where $R_{0}$ represents the rate of background ionization in zerofield limit; $R_{\text {ion }}=\alpha N_{e}\left|w_{e}\right|$ is the rate of electron impact ionization ( $\alpha$ stands for Townsend's ionization coefficient, $\mathrm{m}^{-1}$ ); $R_{\text {att }}=\eta N_{e}\left|w_{e}\right|$ is the rate of electron attachment to electronegative molecules ( $\eta$ is attachment coefficient, $\mathrm{m}^{-1}$ ); and $R_{d e t}=k_{d e t} N_{e} N_{n}$ is the rate of detachment of electron from negative ions $\left(k_{\text {det }}\right.$ is detachment coefficient, $\left.\mathrm{m}^{3} / \mathrm{s}\right)$. Two types of recombination are considered, electron-ion and ion-ion, with the rates $R_{e p}=\beta_{e p} N_{e} N_{p}$ and $R_{p n}=\beta_{p n} N_{p} N_{n}$, respectively $(\beta$ stands for corresponding recombination coefficient, $\mathrm{m}^{3} / \mathrm{s}$ ). Ionization of neutral molecules by photons is a process leading to non-local generation of secondary electrons in gas volume. It is crucial for streamer development in air since it provides seeding electrons in front of streamer head. The rate $R_{p h}$ is obtained by utilizing a differential photo-ionization model [9] within which it is represented as a sum (4) of solutions of a set of Helmholtz equations (5):

$R_{p h}=\sum_{i} R_{p h}^{i}$

$\nabla^{2} R_{p h}^{i}(\mathrm{r})-\left(\lambda_{i} \mathrm{p}_{\mathrm{O}_{2}}\right)^{2} R_{p h}^{i}(\mathrm{r})=-\mathrm{A}_{i} \mathrm{p}_{\mathrm{O}_{2}}{ }^{2} \mathrm{I}(\mathrm{r})$

Here $\mathrm{p}_{\mathrm{O}_{2}}$ represents partial pressure of oxygen in air. The local intensity of generation of photons is a function of electron impact ionization rate $\mathrm{I}(\mathbf{r})=\mathrm{p}_{\mathrm{q}} \xi R_{\text {ion }} /\left(\mathrm{p}_{\mathrm{O}_{2}}+\mathrm{p}_{\mathrm{q}}\right)$, where $\mathrm{p}_{\mathrm{q}}$ is the quenching pressure (60 Torr) and $\xi$ is the efficiency of generation of photons. Three terms Helmholtz approximations is used in the present study and the corresponding coefficients $A_{i}$ and $\lambda_{i}$ in (5) are taken from [9].
The values of the parameters used in the model are summarized in Table 1. Field dependencies of the reduced ionization and attachment coefficients are presented in Fig. 1. Drift velocity of electrons as function of electric field is approximated as $w_{e}=3.2 \cdot 10^{3}(E / N)^{0.8}, \mathrm{~m} / \mathrm{s}$, and the diffusion coefficient is obtained as $D_{e}=7 \cdot 10^{-2}+8 \cdot(E / N)^{0.8}, \mathrm{~m}^{2} / \mathrm{s}$ (here, the reduced field $E / N$ is in $\mathrm{Td}$, and $N$ is air density, $\mathrm{m}^{-3}$ ).

\section{B. Model of Charge Transport in Solid Dielectric Barrier}

In the present study, it is assumed that electrons generated in gas phase are injected into the barrier while reaching its surface. The process is controlled by local electric field and is governed by Schottky's mechanism. Charge injection is dependent on the direction of the normal component of the field electric field on the surface. Thus, electrons are injected if surface field is pointing out of the gas-solid interface and holes are injected in opposite situation. The density of corresponding current is calculated using known expression given e.g. in [10]:

$$
J_{c, a}=A T^{2} \exp \left(-\psi_{c, a} / k T\right) \exp \left(\frac{q}{k T} \sqrt{q E_{c, a} / 4 \pi \varepsilon_{0} \varepsilon_{r}}\right)
$$

Here, subscripts $c$ and $a$ represents virtual cathode and anode (hence indicate alignment of the current with the electric field), respectively; $A=1.2 \cdot 10^{6} \mathrm{~A} /\left(\mathrm{m}^{2} \mathrm{~K}^{2}\right)$ is Richardson's constant; $T$ is absolute temperature, $\mathrm{K} ; \psi$ is the injection energy barrier, $\mathrm{eV} ; k$ stands for Boltzmann's constant.

Once the charges are inside the solid material, they are set in motion by local fields. The mobility of charge carriers is approximated as $\mu_{e, h}=\mu_{b e, h} \exp \left(-\psi_{s t e, h} / \mathrm{kT}\right)$ and the

TABLE I. PARAMETERS USED IN THE SIMULATIONS

\begin{tabular}{|l|c|}
\hline \multicolumn{1}{|c|}{ Parameter } & Value \\
\hline$\mu_{p}, \mathrm{~m}^{2} / \mathrm{Vs}$ & $2 \cdot 10^{-4}$ \\
\hline$D_{p}, \mathrm{~m}^{2} \mathrm{~s}^{-1}$ & $5.05 \cdot 10^{-6}$ \\
\hline$\mu_{n}, \mathrm{~m}^{2} / \mathrm{Vs}$ & $2.2 \cdot 10^{-4}$ \\
\hline$D_{n}, \mathrm{~m}^{2} \mathrm{~s}^{-1}$ & $5.56 \cdot 10^{-6}$ \\
\hline$\beta_{e p}, \mathrm{~m}^{3} \mathrm{~s}^{-1}$ & $5 \cdot 10^{-14}$ \\
\hline$\beta_{p n}, \mathrm{~m}^{3} \mathrm{~s}^{-1}$ & $2.07 \cdot 10^{-12}$ \\
\hline$R_{0}, \mathrm{~m}^{-3} \mathrm{~s}^{-1}$ & $1.7 \cdot 10^{9}$ \\
\hline$k_{\text {det }}, \mathrm{m}^{3} \mathrm{~s}^{-1}$ & $10^{-18}$ \\
\hline
\end{tabular}



Fig. 1. Reduced ionization $\alpha / N$ and attachment $\eta / N$ coefficients as functions of the reduced electric field. 
diffusion coefficient can be determined from Einstein's relation $D_{e, h}=\mathrm{kT} / \mathrm{q} \mu_{e, h}$. In the last two expressions, subscripts $e$ and $h$ represents electrons and holes respectively; $\mu$ is band mobility, $\mathrm{m}^{2} / \mathrm{Vs} ; \psi_{\mathrm{st}}$ is the energy needed to overcome shallow traps, eV. Single trap level is considered for the charge carriers. Hence, the total flux is defined as

$\mathbf{q}_{e, h}=\operatorname{sign}_{e, h} n_{e, h} \mu_{e, h} \mathbf{E}-D_{e, h} \nabla n_{e, h}$

where $n$ stands for charge carrier density, $\mathrm{m}^{-3}$, and sign represents polarity of the charge. Further, the continuity condition for the total flux can be written as

$\partial n_{e, h} / \partial \mathrm{t}+\nabla \cdot\left(\mathbf{q}_{e, h}\right)=\mathrm{R}_{e, h}$

where the term $R_{e, h}$ is the net rate of processes associated with charge transport. In the analysis, trapping/release in/from local states and recombination at centers created by deeply trapped charges are considered. The respective rates for electrons: $R_{\text {tre }}=T_{e} n_{e}\left(1-n_{e t} / n_{0 e t}\right), R_{\text {detre }}=v \exp \left(-\psi_{\text {et }} / k T\right) n_{\text {et }}^{2} / n_{0 e t}$, $R_{\text {rece }}=\beta_{\text {eht }} n_{e} n_{h t} ;$ and for holes $R_{t r h}=T_{h} n_{h}\left(1-n_{h t} / n_{0 h t}\right)$, $R_{\text {detrh }}=v \exp \left(-\psi_{h t} / k T\right) n_{h t}^{2} / n_{0 h t}, R_{\text {rech }}=\beta_{\text {eth }} n_{h} n_{\text {et }}$. Thus, the net rates are defined as

$R_{e}=-R_{\text {rece }}-R_{\text {tre }}+R_{\text {detre }}$

$R_{h}=-R_{\text {rech }}-R_{\text {trh }}+R_{\text {detrh }}$

Here: $n_{e t}$ and $n_{h t}$ are densities of trapped electrons and holes; $n_{0 e t}$ and $n_{0 h t}$ are densities of deep traps for electrons and holes respectively (assumed to be uniformly distributed); $\beta_{\text {eht }}$ and $\beta_{\text {eth }}$ are recombination coefficients (mobile electrons - trapped holes and mobile holes - trapped electrons, respectively); $T_{e}$ and $T_{h}$ stand for trapping coefficients, $\mathrm{s}^{-1} ; v$ is attempt to escape frequency, $\mathrm{s}^{-1} ; \psi_{\mathrm{et}}$ and $\psi_{\mathrm{ht}}$ indicate barrier height for de-trapping of electrons and holes, eV. The dynamics of filling deep traps is described by the following equations:

$d n_{\text {et }} / d t=R_{\text {tre }}-\mathrm{R}_{\text {rech }}-\mathrm{R}_{\text {detre }}$

$d n_{e t} / d t=R_{\text {trh }}-\mathrm{R}_{\text {rece }}-\mathrm{R}_{\text {detrh }}$

Similarly to the model of charge transport in gas, the local charges are coupled to the electric field via Poisson's equation

$\nabla \cdot\left(\varepsilon_{0} \varepsilon_{\mathrm{r}} \Delta V\right)=-q\left(n_{h}+n_{h t}-n_{e}-n_{e t}\right)$

Note that the space charge in solid phase is determined by mobile and trapped electrons and holes.

Parameters of the processes in solid material used in the simulations are presented in Table 2 and correspond to typical properties of polyethylene.

\section{Coupling Between the Two Models}

The coupling between charge transport models in two media is implemented by introducing current conservation equation at solid surface incorporating different mechanism of charge transport:

$d \sigma_{s} / d t=-j_{s}-j_{b}-j_{g}$

Here $\sigma_{S}$ is surface charge density on gas-solid interface, $\mathrm{C} / \mathrm{m}^{2}$. The rate of surface charge accumulation/decay is defined by a sum of current densities due to surface conduction $j_{\mathrm{s}}$ and bulk conduction $j_{\mathrm{b}}$ in solid as well as due to conduction through gaseous phase $j_{\mathrm{g}}$.
TABLE II. PARAMETERS USED IN THE SIMULATIONS OF CHARGE TRANSPORT IN SOLID MATERIAL

\begin{tabular}{|l|l|}
\hline \multicolumn{1}{|c|}{ Parameter } & \multicolumn{1}{c|}{ Value } \\
\hline Band mobilities, $\mathrm{m}^{2} / \mathrm{Vs}$, electrons $/$ holes & $10^{-14} / 2 \cdot 10^{-13}$ \\
\hline Depths of shallow traps, eV & 0.05 \\
\hline Recombination coefficients, $\mathrm{m}^{3} / \mathrm{s}, R_{\text {eht }}=R_{\text {eth }}$ & $6.4 \cdot 10^{-22}$ \\
\hline Densities of deep traps, $\mathrm{m}^{-3}$, electrons $/$ holes & $6.3 \cdot 10^{20}$ \\
\hline Trapping coefficients, $\mathrm{s}^{-1}$, electrons / holes & $10^{-1} / 2 \cdot 10^{-1}$ \\
\hline Attempt-to-escape frequency, $\mathrm{s}^{-1}$ & $6 \cdot 10^{12}$ \\
\hline Barrier height for de-trapping, eV, electrons / holes & $0.96 / 0.99$ \\
\hline Barrier height for injection, eV, electrons / holes & $1.27 / 1.16$ \\
\hline Material dielectric constant $\varepsilon_{r}$ & 2.3 \\
\hline
\end{tabular}

\section{Simulation Conditions and Model Implementation}

The presented model was utilized for analyzing streamer development in needle-plane electrode system containing solid floating barrier as can be seen in Fig. 2. The needle radius is $0.5 \mathrm{~mm}$ and the separation between the electrodes $50 \mathrm{~mm}$. The barrier with thickness of $1 \mathrm{~mm}$ and radius $50 \mathrm{~mm}$ is located at a distance of $1 / 3$ of the inter-electrode gap $(7 \mathrm{~mm})$ from the needle tip. A stepped positive voltage of $60 \mathrm{kV}$ is applied to needle electrode. The plate electrode is grounded. The dimensionality of the problem was reduced to $2 \mathrm{~d}$ utilizing axial symmetry. Boundary conditions for the gas domain at the electrodes include outflow flux for species reaching metallic surfaces and zero flux for the carriers flow in opposite direction. Zero flux condition is provided on the lateral boundaries. As for the solid barrier, the fluxes of electrons and holes are defined by (6). Equation (12) is solved on the gassolid interfaces. For Poisson's equation, Dirichlet conditions are used specifying potentials for the electrodes as described above while Neumann condition is applied on external boundaries.

The set of coupled equations was implemented and solved in Comsol Multiphysics software using customized logarithmic formulation. For time stepping, an implicit algorithm based on backward differential formula was used. The segregation of variables was utilized by splitting them into three groups. Iterative solver was employed for solving the model.

\section{RESULTS AND DISCUSSION}

Computed evolution of electron density during streamer initiation and propagation is shown Fig. 2. As the initial electric field is highest next to the needle electrode, formation of an electronic cloud is observed here almost immediately after voltage application. The discharge region expands towards the cathode (plane) very quickly. At the instant $0.2 \mathrm{~ns}$ when the discharge front approaches the barrier, the peak density of electrons is $\sim 10^{15} \mathrm{~cm}^{-3}$. After that, the streamer channel starts propagating along the surface of the barrier and charge deposition on the other side (facing to the plane) can also be observed due to induced field. At $29 \mathrm{~ns}$ the expanding discharge reaches the edge of the barrier. Rather than continuing growing on the other side of the barrier to the central axis or following the shortest path to the ground electrode, the streamer moves practically along the electric field line and finally bridges the gap at $49 \mathrm{~ns}$. 
The electric field distribution at $10 \mathrm{~ns}$ is plotted in Fig.3. As seen, it is characterized by strong enhancement at the front of the discharge propagating along the surface where the peak value reaches $\sim 75 \mathrm{kV} / \mathrm{cm}$. The field is weak in other regions of the domain especially those occupied by charged species where conductivity reaches $\sim 100 \mathrm{mS} / \mathrm{m}$. The average propagation velocity of the discharge is $\sim 2 \mathrm{~mm} / \mathrm{hs}$ that is consistent with experimental observations. The resulting plasma channel appearing after discharge bridges the gap may lead to development of a spark causing a complete breakdown in the system. The obtained discharge trajectory provides an insight for the choice of characteristic path for evaluating breakdown strength with simplified streamer criteria based on space/surface charge free field distribution.
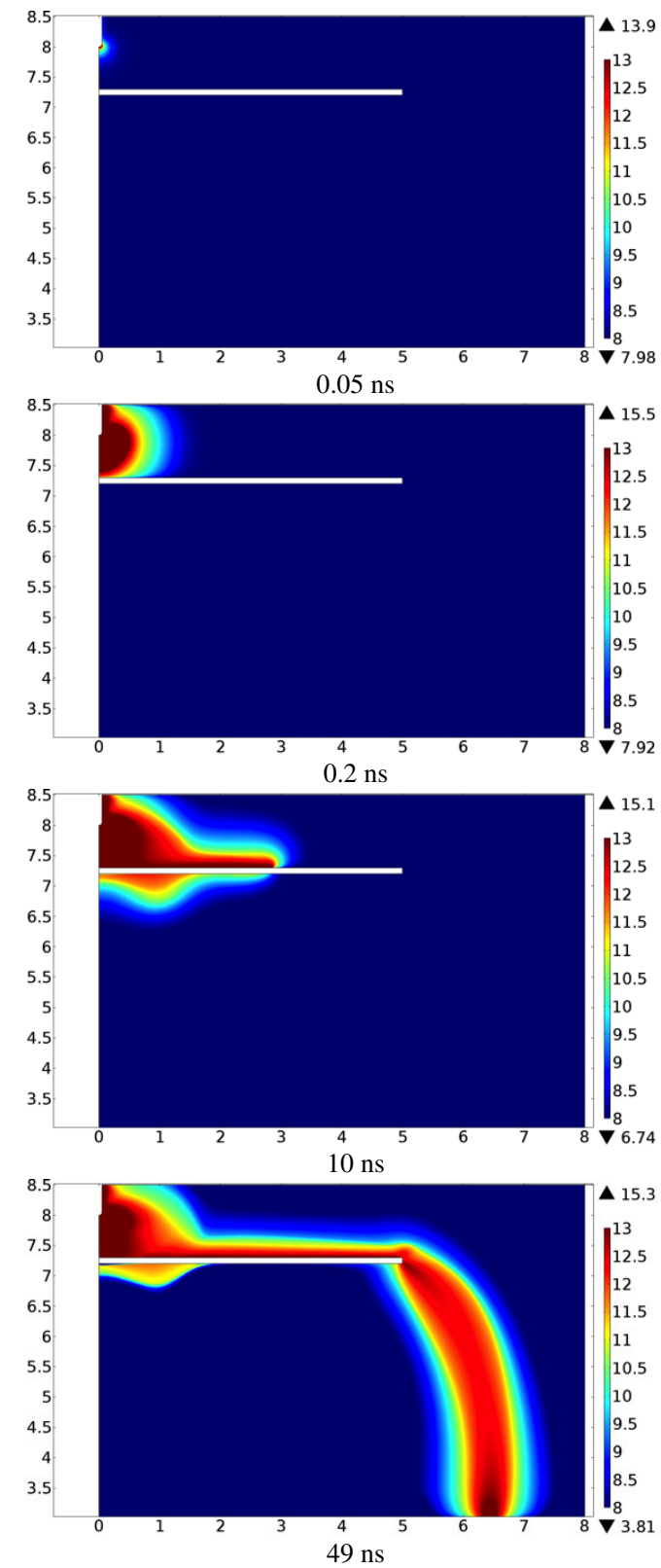

Fig. 2 Distributions of electron density $\left(\mathrm{cm}^{-3}\right.$, logarithmic scale) at different instants after voltage application.

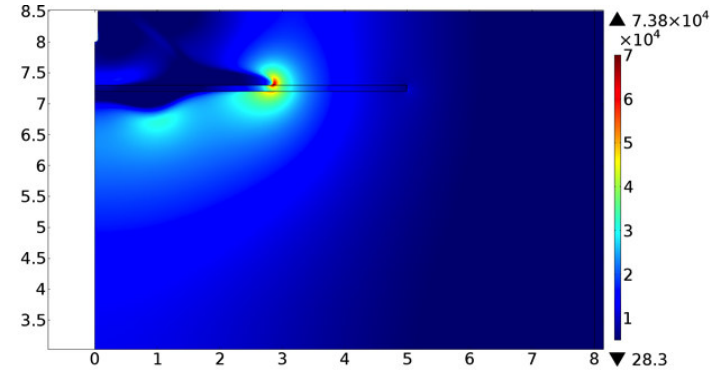

Fig. 3 Electric field $(\mathrm{V} / \mathrm{cm})$ at $10 \mathrm{~ns}$ after voltage application.

\section{CONCLUSION}

A model of streamer discharge in hybrid gas-solid insulation system has been developed and implemented in commercial software utilizing customized formulation of driftdiffusion approximation. The model describes coupled charge transport in gas and solid media as well as on interfaces between them. Simulations of streamer propagation in air in needle-plane electrode system containing solid barrier have demonstrated that the model is capable to reproduce discharge development in gas and along the barrier and yields discharge parameters (field at the front, velocity, conductivity of the channel) consistent with results of other investigations. The conducted study provides knowledge needed for developing design rules for hybrid high voltage insulation.

\section{REFERENCES}

[1] M. Ramesh et al., "Application of streamer criteria for calculations of flashover voltages of gaseous insulation with solid dielectric barrier", Proc. 18th Int. Symp. High Voltage Eng., Aug. 25-30, 2013, Seoul, Korea, pp. 1258-1263.

[2] J. M. Alison, J. V. Champion, S. J. Dodd and G. C. Stevens, "Dynamic bipolar charge recombination model for electroluminescence in polymer based insulation during electrical tree initiation", J. Phys. D: Appl. Phys. Vol. 28, pp. 1693-1701, 1995.

[3] S. Le Roy et al., "Description of charge transport in polyethylene using a fluid model with a constant mobility: fitting model and experiments", J. Phys. D: Appl. Phys. Vol. 39, pp. 1427-1436, 2006.

[4] K. Wu and L. A. Dissado, "Model for electrical tree initiation in epoxy resin”, IEEE Trans. Dielec. Electr. Insul., Vol. 12, pp. 655-668, 2005.

[5] S. Singh, Y. Serdyuk and R. Summer, "Adaptive numerical simulation of streamer propagation in atmospheric air", Proc. 2013 COMSOL Conference, Rotterdam, Netherlands, 2013.

[6] S. Singh, Y. Serdyuk and R. Summer, "Streamer propagation in air in non-axially symmetric electric field", Proc. $19^{\text {th }}$ Int. Symp. High Voltage Eng., 2015, Pilsen, Czech Republic.

[7] M. Sjoberg, Y. V. Serdyuk, S. M. Gubanski and M. A. S. Leijon, "Experimental study and numerical modelling of a dielectric barrier discharge in hybrid air-dielectric insulation", Jour. Electrostatics, Vol. 59, pp. 87-113, 2003.

[8] Y. V. Serdyuk and S. M. Gubanski, "Computer modeling of interaction of gas discharge plasma with solid dielectric barriers", IEEE Trans. Dielec. Elect. Insul., Vol. 12, No. 4, pp. 725-736, 2005.

[9] A. Bourdon et al., "Efficient models for photoionization produced by non-thermal gas discharges in air based on radiative transfer and the Helmholtz equations," Plasma Sour. Sci. Techn., Vol. 16, pp. 656-678, 2007.

[10] F. Baudoin, S. Le Roy, G. Teyssedre and C. Laurent, "Bipolar charge transport model with trapping and recombination: an analysis of the current versus applied electric field characteristic in steady state conditions", J. Phys. D: Appl. Phys. Vol. 41, p. 025306 (10pp), 2008. 